\title{
Design for a New "Dipole-sorter" for Direct and Dose Effective Magnetic Dipole Measurement
}

Gianluca Ruffato ${ }^{1}$, Enzo Rotunno ${ }^{2}$ and Vincenzo Grillo ${ }^{3}$

${ }^{1}$ CNR-NANO, Padova, Veneto, Italy, ${ }^{2}$ CNR-NANO, Modena, Emilia-Romagna, Italy, ${ }^{3}$ CNR - Istituto Nanoscienze, Modena, Emilia-Romagna, Italy

One of the most innovative ideas in recent electron optics is the so-called Orbital Angular Momentum (OAM) sorter [1][2]. This device is based on two confocal phase elements that strongly violate the cylindrical symmetry. In the stationary phase approximation, the first element produces a conformal mapping of the wavefunction between polar and Cartesian coordinates. The second element compensates for the transformation phase leaving only the input phase but remapped in the new coordinate system. Thanks to this behavior, the OAM sorter transforms an azimuthal phase gradient into a linear one, therefore being able to perform a map between angular momentum and linear momentum states and allowing for their efficient measurement.

In this contribution we consider a more general class of conformal transformations recently described in optics [4] and demonstrate how to introduce them in electron microscopy trough electrostatic elements [5]. For a given $n$, the new conformal mapping transforms a circle in the plane into a circular sector in the destination plane (Fig. 1(a)), according to the relation, , where $a$ and $b$ are free scaling parameters selected accordingly to the size of the system. This transformation is generated by a phase element of the type , where $m=1-1 / n$ and, being $k$ the wavevector. The second element has substantially the same functional dependence, after the substitution $m=1-n$. A combination of those elements has been demonstrated to perform multiplication and division of OAM beams in a compact and effective manner [4] (Fig. 1(b)). However, they provide further findings and implications going beyond that initial scope.

As a matter of fact, the similarity of their phase function with multipole fields suggests the possibility to implement those elements in an electrostatic way, as already shown for the OAM sorter. This is a general property of conformal mappings to be implemented with functions that are harmonic and therefore can have electrostatic counterparts. We will demonstrate how the desired elements can be generated by proper distributions of charges or currents. Moreover, similarly to the OAM sorter that can map vortex beams (with phase ) to separate positions, the new transformation is demonstrated to efficiently map multipole fields as , where $q=-1 / m$, into a point with position depending on the multipole strength and orientation (Fig. 2) [5].

Considering the importance of multipolar phase for example in nanoparticle magnetism, we believe we have discovered a new convenient, fast and effective way to quickly measure multipole moments with a single measurement capable to analyze small magnetic multipoles with few electrons.

These data are obtained from QSORT personnel. This work is supported by Q-SORT, a project funded by the European Union's Horizon 2020 Research and Innovation Program under grant agreement No. 766970 . 


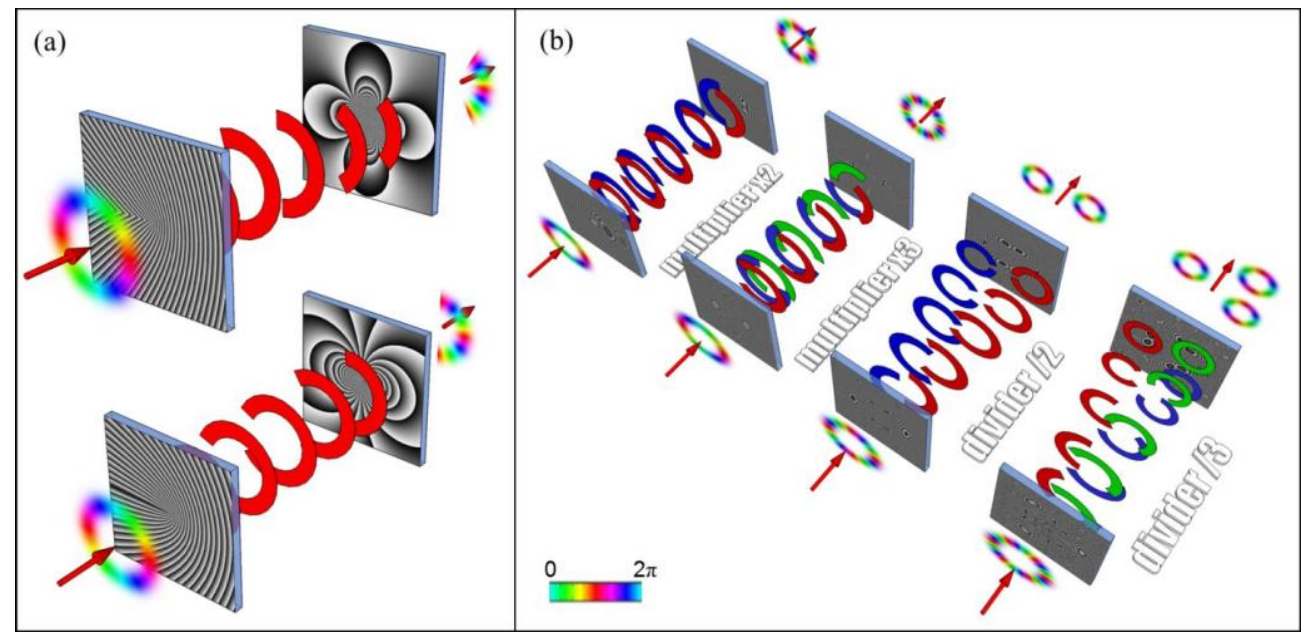

Figure 1. (a) Scheme of 2-fold and 3-fold circular-sector transformations applied to an input vortex wavefunction with orbital angular momentum (OAM) equal to 2. It is worth noting the similarity of the phase elements with integer and fractional multipole fields. (b) Scheme of OAM multiplication and division with combined circular-sector transformations [4].
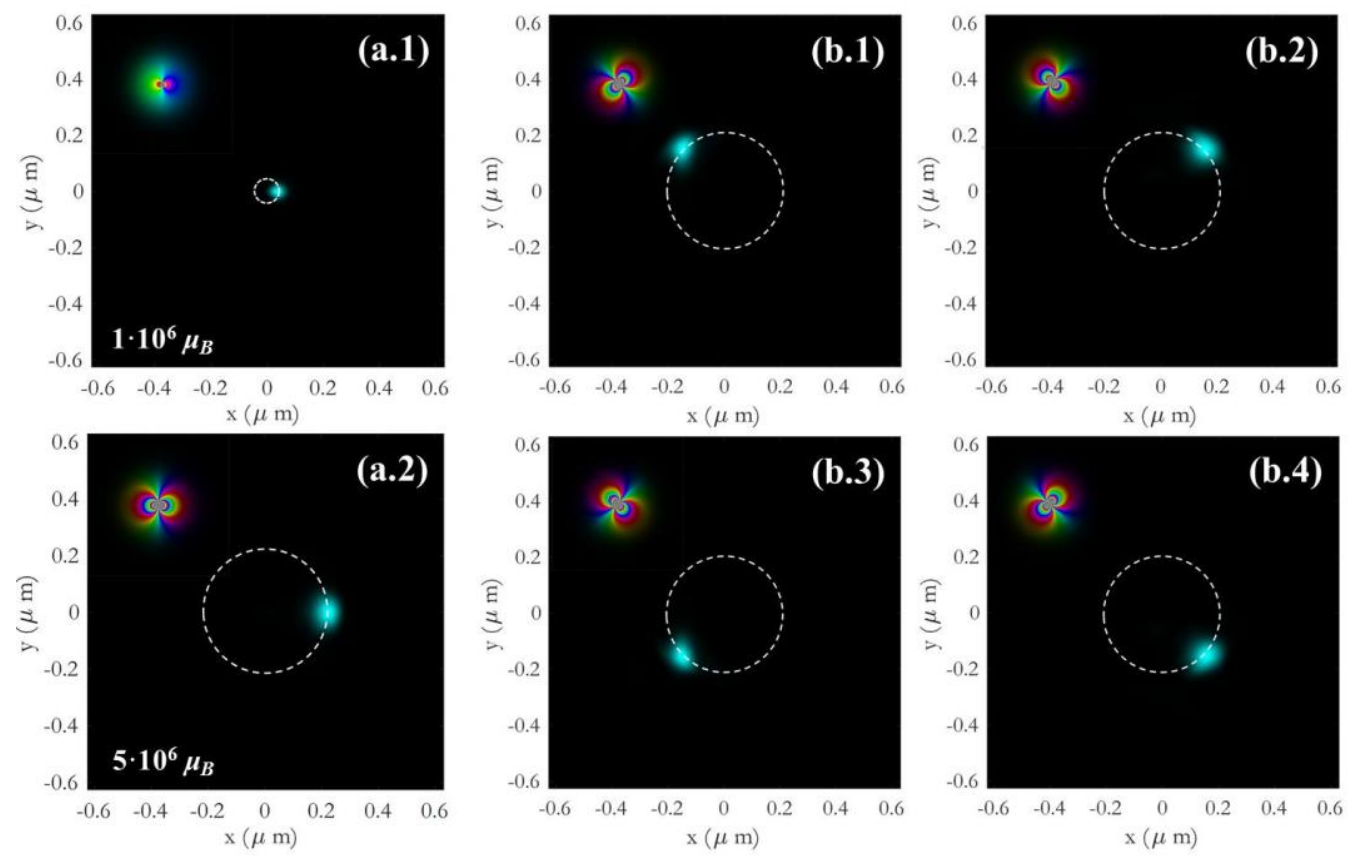

Figure 2. Numerical simulations of dipole sorting using a sequence of circular-sector transformations. The input dipole field is transformed into a far-field spot with position depending on the dipole strength (a.1, a.2) and orientation (b.1-b.4). In each subfigure, the inset plot in the upper left corner shows the input dipole field, where brightness and colours refer to intensity and phase, respectively.

\section{References}

[1] V. Grillo, A. H. Tavabi et al. Nat. Comm. 8, 15536 (2017).

[2] B. McMorran, T.R. Harvey, M.P.J. Lavery, New Journal of Physics 19, 023053 (2017).

[3] AH Tavabi, P Rosi et al. arXiv preprint arXiv:1910.03706

[4] G. Ruffato, M. Massari, and F. Romanato, Light: Science \& Applications 8, 113 (2019).

[5] G. Ruffato, E. Rotunno, V. Grillo, under preparation. 